\title{
FRZB knockdown upregulates $\beta$-catenin activity and enhances cell aggressiveness in gastric cancer
}

\author{
SHUAI QIN $^{1^{*}}$, ZHUO ZHANG ${ }^{2 *},{\text { JIANFANG } \text { LI }^{3} \text { and LU ZANG }}^{2}$ \\ Departments of ${ }^{1}$ Intensive Care Unit and ${ }^{2}$ Surgery; ${ }^{3}$ Shanghai Institute of Digestive Surgery, \\ Ruijin Hospital, Shanghai Jiao Tong University School of Medicine, Shanghai 200025, P.R. China
}

Received January 10, 2014; Accepted March 7, 2014

DOI: $10.3892 /$ or.2014.3109

\begin{abstract}
Studies have shown that FRZB correlates with gastric tumorigenicity and may play role in regulating the Wnt/ $\beta$-catenin signaling pathway. In the present study, we investigated the correlation between FRZB and the Wnt/ $\beta$-catenin signaling pathway using gastric cancer tissues and an FRZB-knockdown gastric cancer cell line model. The protein levels of FRZB and $\beta$-catenin were examined using immunohistochemical staining. FRZB-specific shRNAs were used to generate FRZB-knockdown MKN45 gastric cancer cells. Cell proliferation assay, suspending culture and Annexin V/PI double staining analysis were used to investigate the role of FRZB knockdown in cell growth. In vitro migration/invasion assays were performed. The expression of Wnt/ $\beta$-catenin downstream targets was analyzed by RT-PCR. FRZB mRNA levels showed negative correlation with $\beta$-catenin levels in paired non-tumor and tumor tissues. FRZB protein levels were negatively correlated with $\beta$-catenin levels analyzed by IHC staining. Furthermore, high FRZB protein levels were correlated with membrane localization of $\beta$-catenin. FRZB knockdown increased gastric cancer cell growth in monolayer and soft agar culture; it increased cell aggregates in suspending culture and rendered less apoptosis which indicated increased anti-anoikis growth. FRZB knockdown increased cell migration and invasion and increased the expression of $\mathrm{Wnt} / \beta$-catenin downstream targets such as MMP7 and cyclin D1. Our studies revealed that FRZB levels were correlated with $\beta$-catenin subcellular localization. Knockdown of FRZB in gastric cancer cells increased cell growth and migration/invasion which was also accompanied by activation of $\mathrm{Wnt} / \beta$-catenin downstream targets. FRZB
\end{abstract}

Correspondence to: Professor Lu Zang, Department of Surgery, Ruijin Hospital, Shanghai Jiao Tong University School of Medicine, 197 Ruijin Er Road, Shanghai 200025, P.R. China

E-mail: zanglu@yeah.net

*Contributed equally

Key words: FRZB, $\beta$-catenin, gastric cancer, migration knockdown may upregulate the $\mathrm{Wnt} / \beta$-catenin pathway and promote aggressiveness in gastric cancer.

\section{Introduction}

Gastric cancer is one of the most common causes of cancerrelated mortality worldwide (1). In China, the incidence rate of gastric cancer ranks the third highest amongst the most common cancers (2). Wnt/ $\beta$-catenin signaling is one of the major pathways in gastric carcinogenesis. When Wnt binds to the cell surface receptor Frizzled and activates disheveled, GSK $3 \beta$ is dissociated from their complex. As a result, free $\beta$-catenin accumulates and translocates into the nucleus and subsequently binds to T-cell factor, initiating transcription of its target genes which may be relevant for tumor development and progression (3). These target genes include myelocytomatosis viral oncogene homolog (c-myc) and cyclin D1, matrix metalloproteinase 7 (MMP7), CD44. Epithelial-to-mesenchymal transition is another phenotype caused by $\beta$-catenin activation, which gives cancer cells migration ability accompanied by EMT marker alterations, such as downregulation of epithelial marker E-cadherin and upregulation of mesenchymal markers such as N-cadherin, MMP2 and MMP9 (4). This activation of $\beta$-catenin as a transcriptional factor is the major requirement for cancer initiation in cancer triggered by aberrant Wnt signaling. Hence, more attention has been paid to the regulation of $\beta$-catenin in cancer studies.

$\mathrm{Wnt} / \beta$-catenin can be regulated in multiple steps. One of the key steps in deciding the activation of $\beta$-catenin is the destruction complex APC/Axin/GSK $3 \beta$. The role of this complex is to degrade $\beta$-catenin and prevent its nuclear translocation. In addition to intracellular regulation, outside signals are other regulators of $\mathrm{Wnt} / \beta$-catenin signaling. Secreted inhibitors are other regulators of Wnt signaling including Wnt inhibitory factor (WIF), secreted forms of frizzled proteins (sFRPs) and Dickkopf family proteins (DKKs). FRZB, also known as sFRP3, is one of the Wnt signaling pathway regulators. FRZB contains a 24-amino acid putative transmembrane segment (5), a cysteine-rich domain (CRD) which is similar to the putative Wnt-binding region of the frizzled family of transmembrane receptors, a netrin-like domain (NTR) which is homologous with tissue inhibitors of metalloproteases (TIMPs) (6). Polymorphisms in the FRZB gene have been associated with osteoarthritis (7) 
and are considered one of the osteoblast regulatory genes. FRZB affects the cartilage integrity as well as cortical bone thickness and density. The mechanism of this protection can be partly attributed to FRZB suppression of the expression of WNT/ $\beta$-catenin target genes, including genes for MMP3 and cyclooxygenase 2 (COX2) (8). Further study demonstrated that FRZB may bind and inhibit MMP3 proteinase activity through its NTR domain (8).

FRZB is also involved in malignant tumor generation and progression. Deregulation of FRZB is found in bone-originated malignant diseases. Expression of FRZB was also found to be related to bone involvement at diagnosis in myeloma plasma cells (9). Loss of FRZB expression was commonly found in osteogenic sarcoma tissues (10). FRZB was reportedly highly expressed in gastric cancer tissues, especially in intestinal-type and well-differentiated gastric cancer tissues (11). Expression of FRZB suppresses epithelial original prostate cancer cell in vivo growth and progression (12). FRZB can function as a melanoma migration and invasion suppressor by interfering with Wnt5a signaling (13). FRZB decreases growth and invasiveness of fibrosarcoma cells and this inhibition is associated with downregulation of c-Met expression and inhibited Met-mediated signaling (14). These data strongly suggest a tumor suppressor role of FRZB and involvement of FRZB in regulating the Wnt signaling pathway. Here, we examined the expression of FRZB and $\beta$-catenin, a key downstream factor of Wnt signaling, to observe the expression pattern and association of these two proteins. We further investigated the function of FRZB by knockdown of FRZB expression in gastric cancer cells.

\section{Materials and methods}

Gene expression analysis. For gene expression analysis, 111 gastric cancer and 20 normal gastric tissues were collected from Ruijin Hospital. Total RNA was extracted using TRIzol reagent (Invitrogen, Carlsbad, CA, USA), according to the manufacturer's instructions. Gene expression profiling was conducted using U133 plus 2.1 array (Affymetrix, Santa Clara, CA, USA).

Immunohistochemistry. Gastric cancer tissues, confirmed by pathological diagnosis, were obtained from 89 patients who underwent radical resection for gastric cancer between 2006 and 2008 at the Department of Surgery, Ruijin Hospital, Shanghai, China. The corresponding non-tumor gastric tissues were obtained at least $6 \mathrm{~cm}$ from the tumors. All tissue samples were formalin-fixed and paraffin-embedded. TNM staging was classified based on the criteria of the American Joint Committee on Cancer (AJCC, 7th edition) for gastric cancer. The present study was approved by the Shanghai Jiao Tong University Medical School Institutional Review Board.

Immunohistochemistry staining was performed by using a highly sensitive streptavidin-biotin-peroxidase detection system with gastric cancer tissue microarrays. Rabbit monoclonal anti-FRZB (working dilution 1:100) was purchased from LifeSpan Biosciences (Seattle, WA, USA) and Rabbit anti- $\beta$-catenin (working dilution 1:100) was purchased from Cell Signaling (Danvers, MA, USA). Immunolabeling was conducted using Envision+ Rabbit Polymer (Dako Carpinteria,
CA, USA). The slides were counterstained with hematoxylin and coverslipped.

Immunohistochemistry scoring. The histology of the samples was examined by two histopathologists independently without knowing the clinicopathological information. We scored the slides according to a previous publication (15). The percentage of positive tumor cells was assigned to 5 categories: $\leq 5 \%$ (0), $5-25 \%$ (1), $25-50 \%$ (2), $50-75 \%$ (3), and $\geq 75 \%$ (4). $\leq 5 \%$ positive cells were used as the cutoff to define negative tumors. The intensity of immunostaining was scored as: weak (1), moderate (2), and strong (3). The percentage of positivity of tumor cells and staining intensity were multiplied to produce a weighted score for each tumor specimen. The intensity scores were grouped as low (which included scores 0 to +4 ) and high (which included scores +6 and +12 ).

Cell culture. Human gastric cancer cell line MKN45 was obtained from Shanghai Institute of Cell Biology, Chinese Academy of Sciences. The cells were grown in RPMI-1640 medium containing $10 \%$ fetal bovine serum (FBS), penicillin and streptomycin (Gibco-BRL, Gaithersburg, MD, USA).

Cell proliferation and soft agar assay. Cell proliferation was assessed using the Cell Counting Kit-8 (CCK-8; Dojindo Laboratories, Kumamoto, Japan) assay. Soft agar colony formation assay was performed by using $0.3 \%$ agar in complete medium (RPMI-1640 medium containing 10\% FBS) with cells as the feeder layer and $0.6 \%$ agar in complete medium as the bottom layer.

FRZB-specific shRNA and transfection. The sequence used for construct of FRZB-shRNA was 3'-GGAGATTCTAAA GTCCTCTTTCAAGAGAAGAGGACTTTAGAATCTCC-5'. The shRNAs were cloned into pGPU6/Neo (Shanghai GenePharma, Shanghai, China). Plasmids were transfected into gastric cancer cells using Lipofectamine 2000 (Invitrogen). G418 was used for stable clone selection.

$R T-P C R$. RT-PCR was carried out and the set of primers for FRZB was: F, 5'-GAGGAGCTGCCAGTGTACGAC-3' and R, 5'-GAAAATCAGCTCCGTCCGC-3'; GAPDH: F, 5'-GGAC CTGACCTGCCGTCTAG-3' and R, 5'-GTAGCCCAGGAT GCCCTTGA-3' respectively (11). Primers for Ecad were: F, 5'-TTCCCTCGACACCCGATTCA-3' and R, 5'-CCAGAAAC GGAGGCCTGATG-3'; Ncad: F, 5'-CCCGGTTCATTT GAGGGCA-3' and R, 5'- GGCATTGGGATCGTCGCAT-3'; MMP7: F, 5'-GTCTCTGGACGGCAGCTATG-3' and R, 5'-TAGTCCTGAGCCTGTTCCCA-3'; cyclin D1: F, 5'-GATGC CAACCTCCTCAACGA-3' and R, 5'-GGAAGCGGTCCAG GTAGTTC-3'. Relative density of target gene levels were measured by the following equation: Relative density = density of band of target genes/density of GAPDH.

Western blotting. Whole cell lysates were harvested using RIPA cell lysis buffer supplemented with a protease inhibitor cocktail (Sigma). A total of $50 \mu \mathrm{g}$ protein were separated by SDS-polyacrylamide gel electrophoresis and blotted onto $0.22 \mu \mathrm{m}$ polyvinylidene difluoride membranes (Millipore, Billerica, MA, USA). For extracting nuclear protein, nuclear 

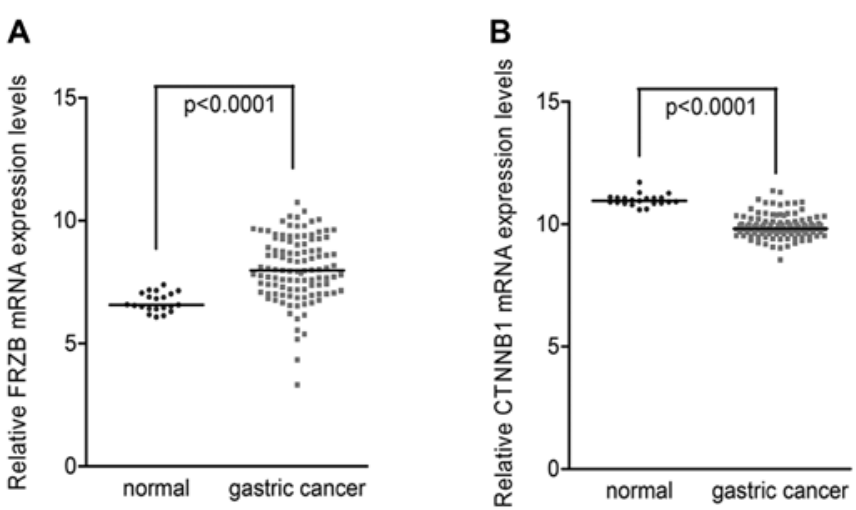

Figure 1. The mRNA levels of (A) FRZB and (B) $\beta$-catenin/CTNNB1 in a 131-sample dataset including 20 normal gastric tissues and 111 gastric cancer tissues.

extract kit (Active Motif, Carlsbad, CA, USA) was used following the manufacturer's instructions. Antibodies against FRZB (LifeSpan) were used at 1:1,000 dilutions. Antibodies against GAPDH (Sigma) were used at a 1:5,000 dilution. Antibodies against Lamin B1 and $\beta$-catenin (Cell Signaling, Boston, MA, USA) were used at 1:1,000 dilutions. The signals were visualized using Odyssey-Sa model 9260 (Li-COR, Lincoln, NE, USA) and images were captured and managed using Odyssey-Sa Infrared Image System (Li-COR).

Cell aggregation assay. Cells were trypsinized in the presence of EDTA, washed twice in PBS and suspended at a concentration of $2.5 \times 10^{5}$ cells $/ \mathrm{ml}$ in DMEM with $10 \%$ FBS. Cell culture dishes (diameter, $10 \mathrm{~cm}$ ) were pre-coated with poly-2-hydroxyethyl-methacrylate (poly-HEMA; Sigma, St. Louis, MO, USA) to block cell attachment. Same amount of MKN45 (parental), vector-only (MKN45/vector) and FRZB-shRNA (MKN45/ FRZB-KD) cells were seeded and cultured for $24 \mathrm{~h}$ (16). Cells were observed using IX71 microscope (Olympus, Tokyo, Japan). Images were captured using Digital Sight DS-U1 (Nikon, Tokyo, Japan) and NIS elements F3.0 software was used (Nikon).

Cell migration and invasion assay. Cell migration was analyzed by a Transwell chamber assay. Cell invasion assays were performed using BioCoat Matrigel Invasion Chambers (BD Biosciences, Franklin Lakes, NJ, USA) and 10\% FBS was used as the chemoattractant. Cells on the lower surface of the insert were fixed and stained followed by counting under a light microscope. Cells were visualized using BX50 microscope (Olympus). Images were captured using Digital Sight DS-U2 (Nikon) and NIS elements F3.0 software was used (Nikon).

Statistical analysis. For IHC staining, the differences in clinicopathological features between the different groups were determined using Pearson's $\chi^{2}$ test. $\mathrm{P}<0.05$ was considered to indicate a statistically significant difference. The significance of differences between experimental groups was analyzed using the Student's t-test and two-tailed distribution. Statistical Package for the Social Sciences version 13.0 (SPSS, Inc., Chicago, IL, USA) was used for all statistical analyses.
Table I. Correlation between FRZB and $\beta$-catenin RNA analyzed using cDNA microarray dataset.

\begin{tabular}{lcc}
\hline Tissues & Corr $_{\text {FRZB- }- \text {-catenin }}$ & P-value \\
\hline Gastric cancer & -0.263 & 0.005 \\
Non-tumor & -0.063 & 0.402 \\
\hline
\end{tabular}

Table II. Association between FRZB and subcellular localization of $\beta$-catenin in gastric cancer tissues.

\begin{tabular}{lccc}
\hline & \multicolumn{2}{c}{ FRZB } & \\
\cline { 2 - 3 }$\beta$-catenin & Low (\%) & High (\%) & P-value \\
\hline Membrane & & & \\
Negative & $47(94.0)$ & $29(72.5)$ & 0.005 \\
Positive & $3(6.0)$ & $11(27.5)$ & \\
Nucleus & & & \\
Negative & $37(74.0)$ & $35(87.5)$ & 0.112 \\
Positive & $13(26.0)$ & $5(12.5)$ & \\
\hline
\end{tabular}

\section{Results}

FRZB levels are negatively correlated with $\beta$-catenin levels in gastric cancer tissues. As demonstrated by previous studies $(3,5)$, FRZB is associated with the activity of $\beta$-catenin. To investigate the correlation between FRZB and $\beta$-catenin, we first analyzed mRNA levels of FRZB and $\beta$-catenin in nontumor and tumor gastric tissues. cDNA microarray dataset using a cohort of 111 gastric cancer tissues and 20 normal gastric mucosal tissues was analyzed. This analysis demonstrated that FRZB mRNA levels were higher in gastric cancer tissues than in non-tumor tissues (Fig. 1A). On the contrary, $\beta$-catenin mRNA levels were lower in gastric cancer tissues than in non-tumor tissues (Fig. 1B). Pearson correlation analysis revealed that the negative correlation between FRZB and $\beta$-catenin levels was statistically significant in gastric cancer tissues (Corr $=-0.263, \mathrm{P}=0.005)$ but not in normal gastric tissues (Corr $=-0.063, \mathrm{P}=0.402$ ) (Table I). These data showed a negative correlation between FRZB and $\beta$-catenin in gastric cancer tissues.

FRZB levels are correlated with $\beta$-catenin localization in gastric cancer tissues. To investigate the possible role of FRZB in regulating the function of $\beta$-catenin, we performed IHC staining using the same cohort of specimens as we used for FRZB staining. We discovered that FRZB was correlated with the sub-cellular localization of $\beta$-catenin, which might suggest the activation of $\beta$-catenin related signaling pathway. Among these 90 cases, 14 cases displayed membrane staining of $\beta$-catenin, while 18 cases showed nuclear $\beta$-catenin. As shown in Table II, membrane $\beta$-catenin tended to exist in the high FRZB expression group (11/14 cases), while nuclear $\beta$-catenin tended to exist in the low FRZB expression group (13/18 cases). The FRZB protein levels were associated with 

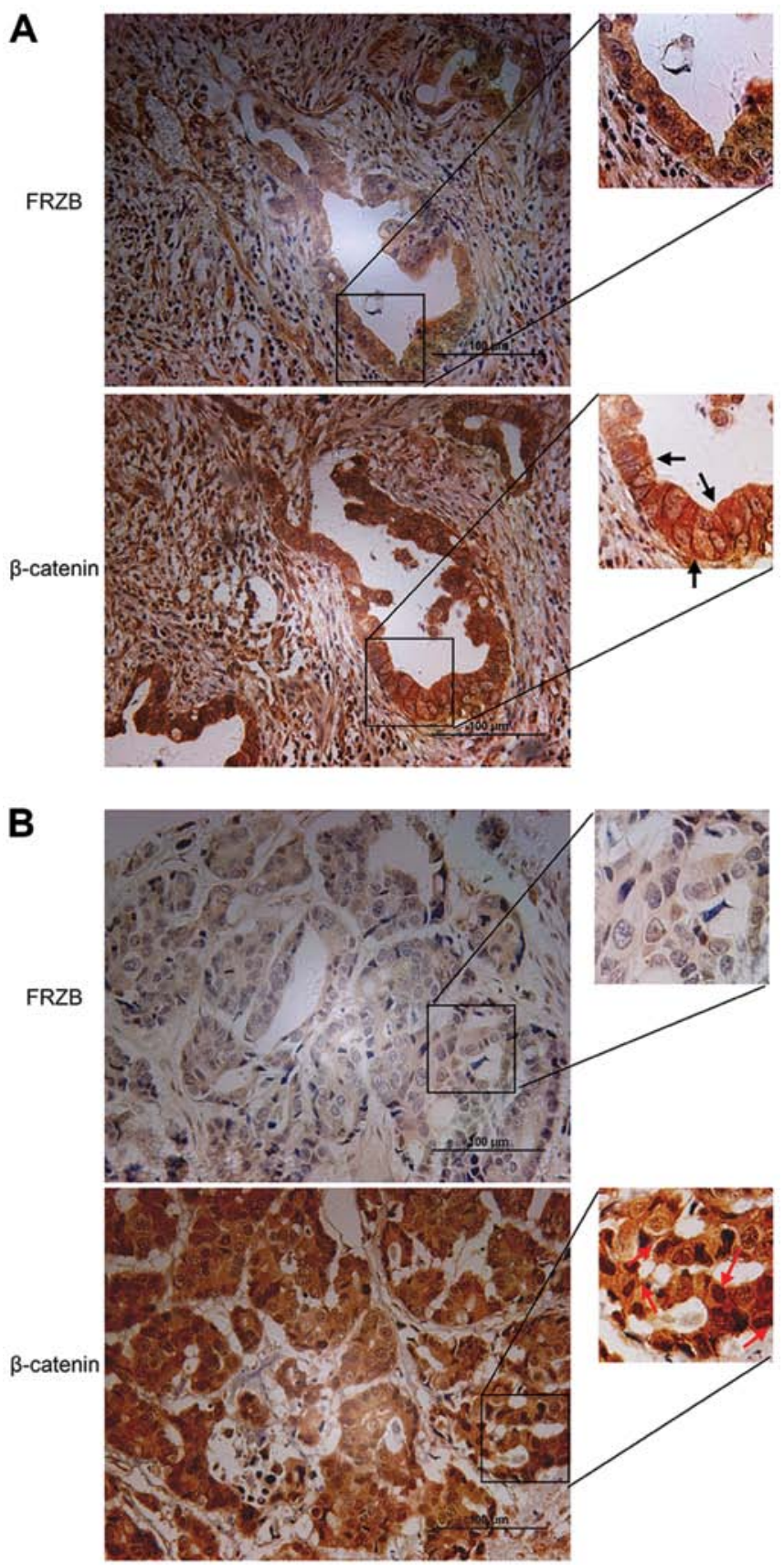

Figure 2. FRZB protein levels are correlated with $\beta$-catenin subcellular localization. (A) A representative sample showing strong FRZB staining (upper) and membrane $\beta$-catenin localization (lower) using IHC staining. Black arrows show membrane staining of $\beta$-catenin. (B) A representative sample showing weak FRZB staining (upper) and nuclear localization of $\beta$-catenin (lower) using IHC staining. Red arrows show nuclear staining of $\beta$-catenin.

$\beta$-catenin membrane location with statistical significance $(\mathrm{P}=0.005)$. Although not statistically significant $(\mathrm{P}=0.112)$, gastric cancers in the FRZB-low group had more nuclear $\beta$-catenin. As shown in Fig. 2A, strong FRZB expression was shown in an intestinal-type gastric cancer specimen (upper left) and membrane $\beta$-catenin was also observed in the same case (upper right, black arrows). Weak FRZB expression was shown in a diffuse-type gastric cancer specimen (lower left) and nuclear $\beta$-catenin translocation was observed in the same case (lower right, red arrows) (Fig. 2B). These data suggested
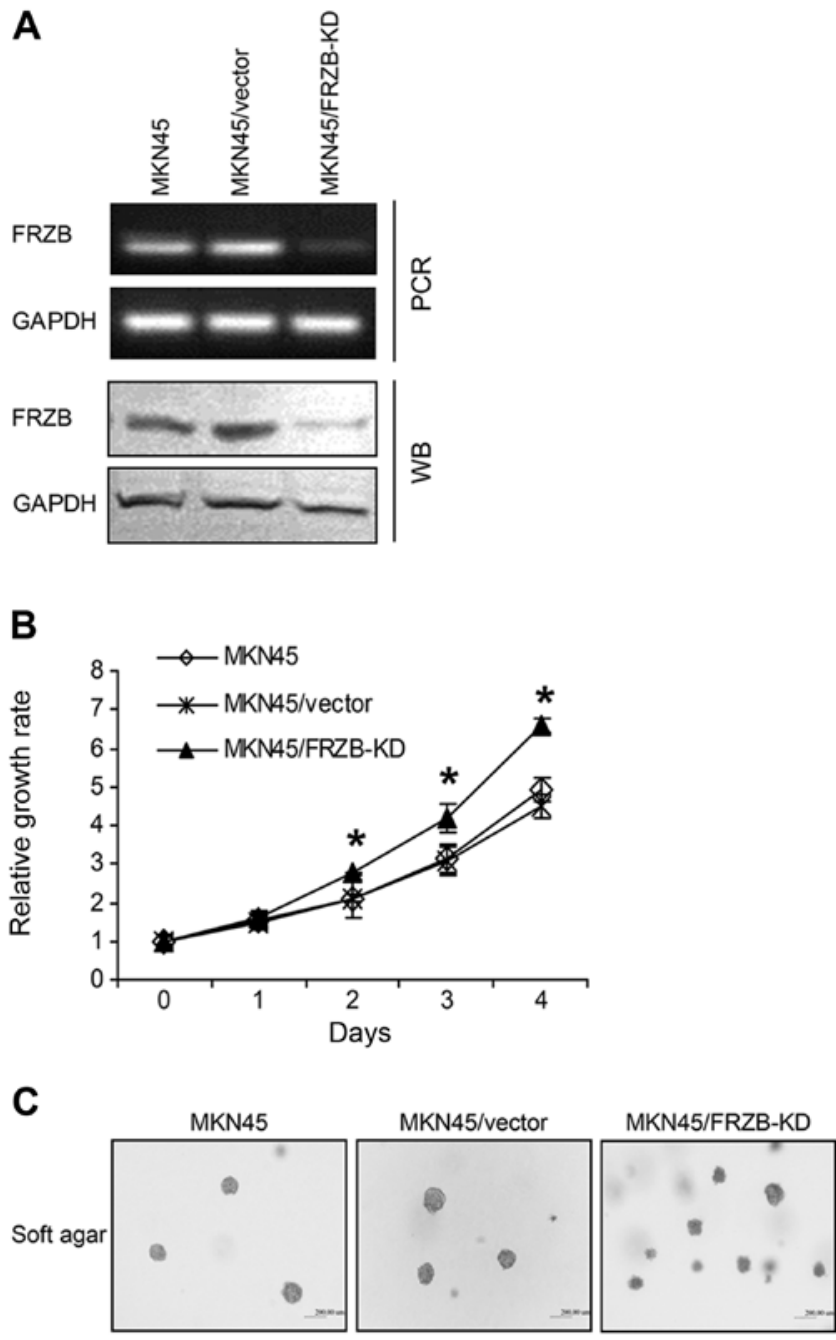

Figure 3. FRZB knockdown in gastric cancer cell increases cell growth (A) FRZB knockdown efficacy in MKN45 cells was examined by RT-PCR and immunoblotting. GAPDH was used as a loading control. (B) Cell proliferation rate in monolayer culture were measured using CCK-8 assays. Parental, vector-only and FRZB-knockdown MKN45 cells were plated in 96-well plates. The cell number was measured at day 0, 1, 2, 3, 4, 5. Growth curves were drawn. (C) Soft agar cell culture was performed to examine the anti-anoikis growth. Representative images are shown at day 14 .

FRZB expression levels were negatively correlated with $\beta$-catenin levels and strongly associated with sub-cellular location of $\beta$-catenin.

FRZB knockdown increases gastric cancer cell growth. Since we observed strong associations between FRZB and $\beta$-catenin in gastric cancer tissue, we next investigated whether the alteration of FRZB levels changed the activity of $\beta$-catenin. Thus, we established an FRZB-knockdown (FRZB-KD) model by stable transfection of FRZB-specific shRNA into gastric cancer cells. FRZB mRNA and protein levels were significantly inhibited by shRNA (Fig. 3A). We first examined the effect of FRZB knockdown on cell growth using CCK-8 analysis. FRZB knockdown significantly increased cell growth rate from day 2 after plating in monolayer culture (Fig. 3B). We then examined whether FRZB-KD had an effect on clonogenicity, using an in vitro assay to investigate tumorigenicity. We performed soft agar culture using parental cells and cells 
A
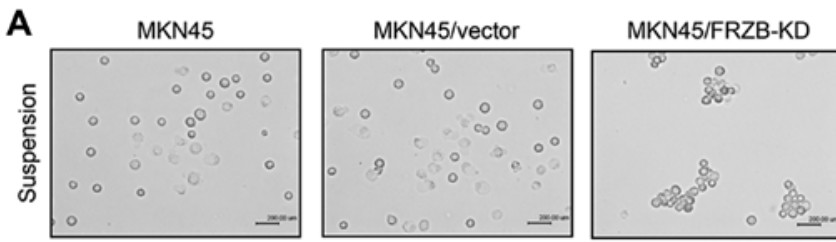

B

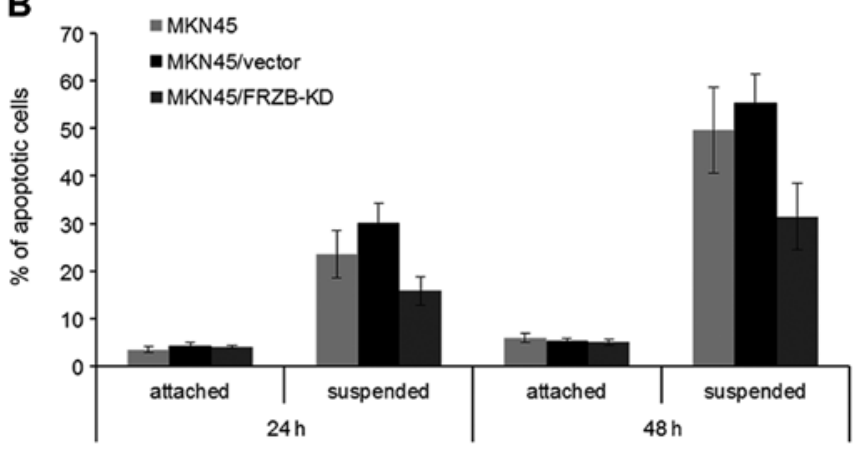

Figure 4. FRZB knockdown in gastric cancer cell increases anti-anoikis growth in suspending culture. (A) FRZB knockdown increased cell aggregation in suspending culture. Representative images are shown. (B) FRZB knockdown decreased cell apoptotic rate in attachment and suspension. Annexin V/PI double staining followed by flow cytometry analysis was performed. Apoptotic rates in parental, vector-only and FRZB-knockdown cells were counted and are shown in plot.

stably transfected with either control-vector or FRZB-shRNAvector. After 14 days of culture, we observed more clones growing in soft agar in FRZB-KD cells than vector control and parental cells (Fig. 3C, upper). These data suggested that FRZB knockdown increased gastric cancer cell growth.

FRZB knockdown increases anoikis resistance in gastric cancer cells. Metastasis is a multi-step process which includes the capacity of detaching from original site, escaping from anoikis and forming secondary tumors (17). We next employed suspension culture assay to examine the anti-anoikis activity, which reflected anchorage-independent growth ability and correlated with metastatic ability of tumor cells. After $24 \mathrm{~h}$ of suspension culture, FRZB-KD cells showed bigger cell aggregation clusters than the other two control groups (Fig. 4A). Apoptosis analysis also showed a lower apoptotic rate in FRZB-KD cells which was consistent with the results from suspension culture (Fig. 4B). These data suggested that anoikis resistance was enhanced by FRZB knockdown.

FRZB knockdown increases migration and invasion in gastric cancer cells. In addition to anoikis, we observed epithelial-to-mesenchymal (EMT) morphological changes in FRZB-KD cells. FRZB-KD cells exhibited scattered clone, while the vector-only and parental cells grew intense clone which showed strong cell interactions (Fig. 5A). We further performed in vitro migration and Matrigel invasion assay to investigate the effect of FRZB-KD on cell motility. As expected, FRZB-KD cells migrated and invaded faster than the other two control cells (Fig. 5B). These data suggested that FRZB knockdown promoted metastatic ability in gastric cancer cells.
A

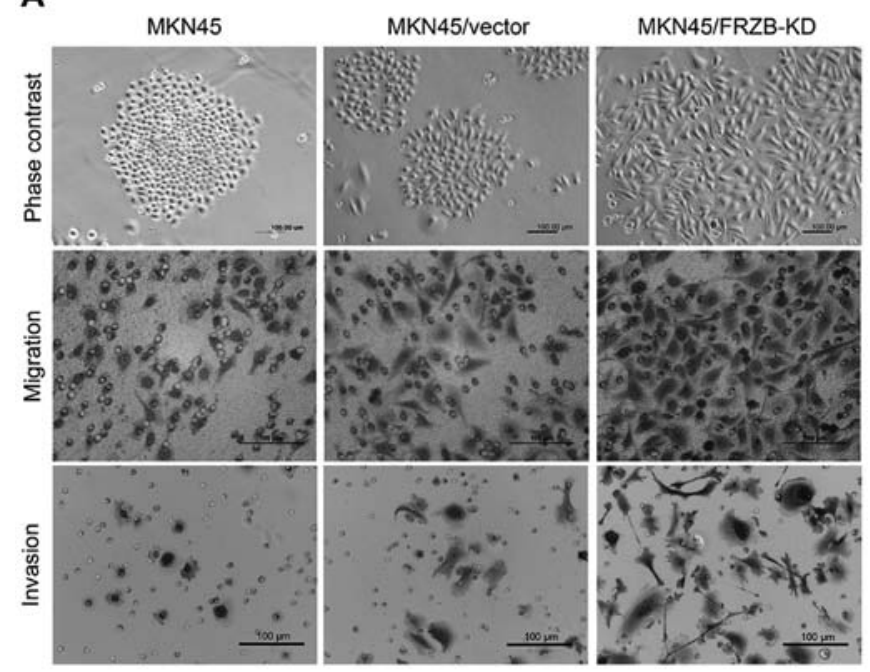

B

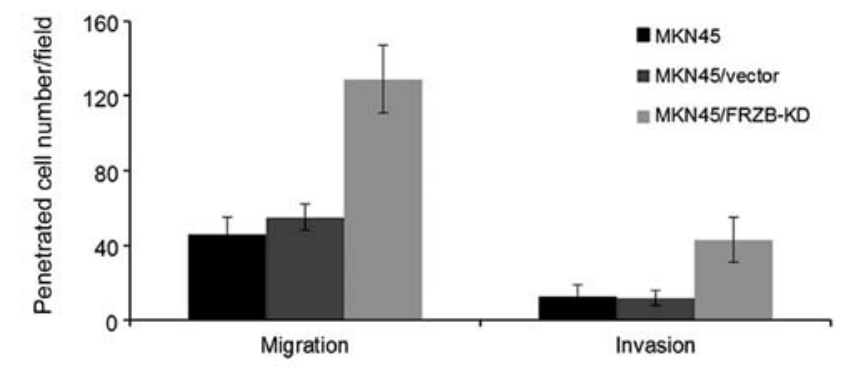

Figure 5. FRZB knockdown in gastric cancer cell increases cell migration and invasion. (A) Representative images showed cell morphology in bright field, migration assay and invasion assay. Original magnification for bright field pictures is $\times 100$. (B) Migrated/invaded cell number per field were calculated and plotted as mean \pm SD.

FRZB knockdown upregulates $\beta$-catenin activity. As we discovered earlier that FRZB and $\beta$-catenin showed negative correlation in gastric cancer tissues, we used an FRZB-KD gastric cancer cell model to further validate this correlation. We first found nuclear $\beta$-catenin level was increased in FRZB-KD cells and total $\beta$-catenin level was also slightly increased (Fig. 6A). We further examined the expression levels of EMT markers and downstream targets which are known to be altered by $\beta$-catenin signaling, such as E-cadherin, $\mathrm{N}$-cadherin, MMP7 and cyclin D1. As expected, levels of the epithelial marker E-cadherin were downregulated while the levels of the mesenchymal marker $\mathrm{N}$-cadherin were upregulated by FRZB knockdown (Fig. 6B). MMP7 and cyclin D1, two direct downstream targets of $\beta$-catenin, were upregulated by FRZB knockdown. Our data indicated activation of $\beta$-catenin signaling in FRZB knockdown gastric cancer cells.

\section{Discussion}

In the present study, we observed a negative concurrence of FRZB levels with $\beta$-catenin subcellular localization in gastric cancer tissues. We further showed a functional correlation of FRZB levels with anoikis and mobility in gastric cancer 
A

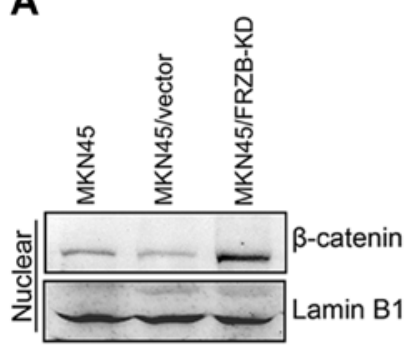

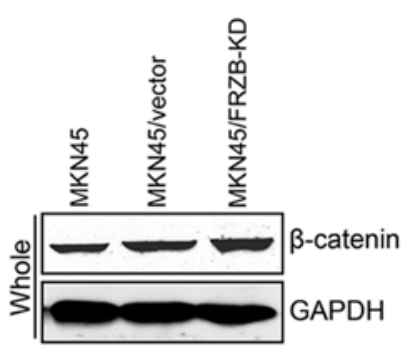

B

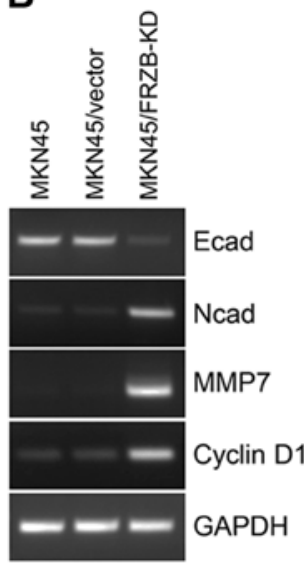

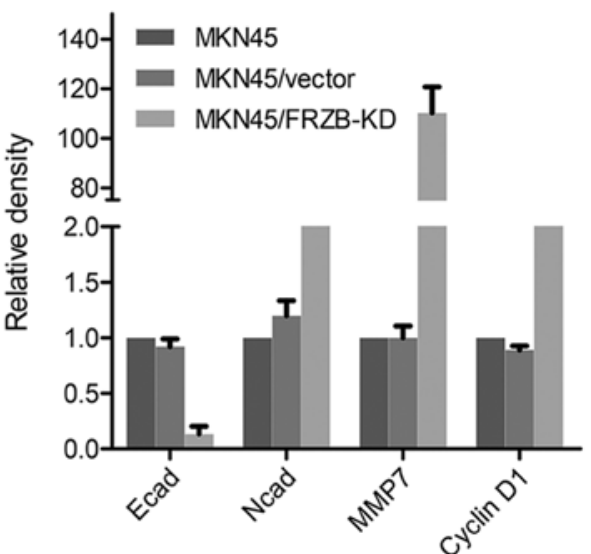

Figure 6. $\beta$-catenin signaling is activated by FRZB knockdown. (A) Levels of nuclear and total $\beta$-catenin were examined in parental, vector-control and FRZB-knockdown MKN45 cells. Lamin B1 was used as a loading control for nuclear extracts and GAPDH was used as a loading control for whole cell extracts. (B) mRNA levels of genes regulated by $\beta$-catenin transcriptional activity were analyzed using PCR. Relative expression fold changes (shown as relative density) are plotted as mean \pm SD.

cells using FRZB knockdown. Furthermore, an activation of Wnt/ $\beta$-catenin downstream targets in FRZB knockdown cells was observed, which is consistent with our findings in gastric cancer tissues.

The various secreted Wnt antagonists interact directly and indirectly to affect Wnt signaling and influence a wide variety of biological processes, including developmental cell fate, differentiation and tumorigenesis. The interactions of Wnt ligands and $\mathrm{Fz}$ receptors are modulated by the secreted Wnt antagonists (sWAs), which can be divided into two functional classes: the soluble frizzled related protein (sFRP) class and the DKK class. Members of the sFRP family include Wnt inhibitory factor-1 (Wif1), sFRP1, 2, 4 and 5 and FRZB (sFRP3). They bind directly to Wnts, thus altering their ability to bind the Fz receptors. Four members of the Dkk family, Dkk1-4, bind to low density lipoprotein receptor related proteins (LRP5 and 6) contained within the Wnt receptor complex and influence Wnt signaling by preventing normal LRP-Fz-Wnt interactions (18). FRZB is one of the classic Wnt signaling inhibitors which have been studied in bone diseases $(19,20)$. In cancer studies, FRZB was found to play a tumor suppressor role in many malignancies including melanoma (13), medulloblastoma (21), gastric cancer (11) and renal cell carcinoma (22). However, there are also studies showing the opposite results. FRZB was found highly expressed in metastatic renal cancer tissues and promoted cell growth, invasion and inhibition of apoptosis (23).
Although FRZB is a member of the secreted Wnt signaling inhibitors and its downregulation has been reported in many malignant tumors, whether FRZB inhibits $\beta$-catenin in gastric cancer remains unclear.

$\beta$-catenin is a multifunctional protein which travels from the cell membrane to the nucleus, thus functioning as an intercellular connecting protein or transcriptional factor. Membrane $\beta$-catenin is part of the cytoskeleton components. Hyperactivation of $\beta$-catenin activity has been reported to play critical role in regulating anoikis resistance in tumor cells (24). As anchorage-independent growth and epithelial-mesenchymal transition are two features associated with anoikis resistance, they are considered to be vital steps during cancer progression and metastatic colonization. Anoikis provides a prerequisite for the dissemination of carcinoma. The ability of cancer cells to resist anoikis is a hallmark of cancer providing tumor cells with metastatic ability. Nuclear accumulation caused activation of $\beta$-catenin was found to increase anoikis in hepatocellular carcinoma cells (25). Further studies revealed direct activation of $\mathrm{Wnt} / \beta$-catenin signaling was the mediator of this phenotype. We also observed larger cell aggregates and less apoptosis in FRZB-knockdown cells under suspension which highly suggested increased anoikis resistance in gastric cancer cells. Downstream of $\beta$-catenin was also activated by FRZB knockdown. Consistent with previous findings, our data suggest that FRZB plays a tumor suppressor role by inhibiting $\beta$-catenin signaling. 


\section{References}

1. Jemal A, Bray F, Center MM, Ferlay J, Ward E and Forman D: Global cancer statistics. CA Cancer J Clin 61: 69-90, 2011.

2. Yang L: Incidence and mortality of gastric cancer in China. World J Gastroenterol 12: 17-20, 2006.

3. Cho KH, Baek S and Sung MH: Wnt pathway mutations selected by optimal beta-catenin signaling for tumorigenesis. FEBS Lett 580: 3665-3670, 2006.

4. Thiery JP, Acloque H, Huang RY and Nieto MA: Epithelialmesenchymal transitions in development and disease. Cell 139: 871-890, 2009.

5. Hoang B, Moos M, Jr., Vukicevic S and Luyten FP: Primary structure and tissue distribution of FRZB, a novel protein related to Drosophila frizzled, suggest a role in skeletal morphogenesis. J Biol Chem 271: 26131-26137, 1996.

6. Banyai L and Patthy L: The NTR module: domains of netrins, secreted frizzled related proteins and type I procollagen $\mathrm{C}$-proteinase enhancer protein are homologous with tissue inhibitors of metalloproteases. Protein Sci 8: 1636-1642, 1999.

7. Killock D: Osteoarthritis: Frzb knockout reveals the complexity of Wnt signaling in joint homeostasis. Nat Rev Rheumatol 8: 123 , 2012.

8. Lories RJ, Peeters J, Bakker A, et al: Articular cartilage and biomechanical properties of the long bones in Frzb-knockout mice. Arthritis Rheum 56: 4095-4103, 2007.

9. Kristensen IB, Haaber J, Lyng MB, et al: Myeloma plasma cell expression of osteoblast regulatory genes: overexpression of SFRP3 correlates with clinical bone involvement at diagnosis. Leuk Lymphoma 54: 425-427, 2013.

10. Mandal D, Srivastava A, Mahlum E, et al: Severe suppression of Frzb/sFRP3 transcription in osteogenic sarcoma. Gene 386: 131-138, 2007.

11. Qu Y, Li JF, Cai Q, et al: Over-expression of FRZB in gastric cancer cell suppresses proliferation and induces differentiation. J Cancer Res Clin Oncol 134: 353-364, 2008.

12. Zi X, Guo Y, Simoneau AR, et al: Expression of Frzb/secreted Frizzled-related protein 3 , a secreted Wnt antagonist, in human androgen-independent prostate cancer PC-3 cells suppresses tumor growth and cellular invasiveness. Cancer Res 65: 9762-9770, 2005.

13. Ekström EJ, Sherwood V and Andersson T: Methylation and loss of Secreted Frizzled-Related Protein 3 enhances melanoma cell migration and invasion. PLoS One 6: e18674, 2011.
14. Guo Y, Xie J, Rubin E, et al: Frzb, a secreted Wnt antagonist, decreases growth and invasiveness of fibrosarcoma cells associated with inhibition of Met signaling. Cancer Res 68: 3350-3360, 2008.

15. Sinicrope FA, Ruan SB, Cleary KR, Stephens LC, Lee JJ and Levin B: bcl-2 and p53 oncoprotein expression during colorectal tumorigenesis. Cancer Res 55: 237-241, 1995.

16. Geiger TR, Song JY, Rosado A and Peeper DS: Functional characterization of human cancer-derived TRKB mutations. PLoS One 6: e16871, 2011.

17. Klein CA:Cancer. The metastasis cascade.Science 321:1785-1787, 2008.

18. Kawano Y and Kypta R: Secreted antagonists of the Wnt signalling pathway. J Cell Sci 116: 2627-2634, 2003.

19. Zhao X, Huang H, Chen Y, et al: Dynamic expression of secreted Frizzled-related protein 3 (sFRP3) in the developing mouse spinal cord and dorsal root ganglia. Neuroscience 248C: 594-601, 2013

20. Kristensen IB, Christensen JH, Lyng MB, et al: Expression of osteoblast and osteoclast regulatory genes in the bone marrow microenvironment in multiple myeloma: only upregulation of Wnt inhibitors SFRP3 and DKK1 is associated with lytic bone disease. Leuk Lymphoma: Aug 5, 2013 (Epub ahead of print)

21. Kongkham PN, Northcott PA, Croul SE, Smith CA, Taylor MD and Rutka JT: The SFRP family of WNT inhibitors function as novel tumor suppressor genes epigenetically silenced in medulloblastoma. Oncogene 29: 3017-3024, 2010.

22. Nikuseva-Martic T, Serman L, Zeljko M, et al: Expression of secreted frizzled-related protein 1 and 3, T-cell factor 1 and lymphoid enhancer factor 1 in clear cell renal cell carcinoma. Pathol Oncol Res 19: 545-551, 2013.

23. Hirata H, Hinoda Y, Ueno K, Majid S, Saini S and Dahiya R: Role of secreted frizzled-related protein 3 in human renal cell carcinoma. Cancer Res 70: 1896-1905, 2010.

24. Lin DC, Zhang Y, Pan QJ, et al: PLK1 is transcriptionally activated by NF- $\kappa \mathrm{B}$ during cell detachment and enhances anoikis resistance through inhibiting beta-catenin degradation in esophageal squamous cell carcinoma. Clin Cancer Res 17: 4285-4295, 2011

25. Fischer AN, Fuchs E, Mikula M, Huber H, Beug H and Mikulits W: PDGF essentially links TGF-beta signaling to nuclear beta-catenin accumulation in hepatocellular carcinoma progression. Oncogene 26: 3395-3405, 2007. 